ÉGYPTE monde arabe

\section{Égypte/Monde arabe}

15-16 | 1993

Les crises soudanaises des années 80

\title{
L'agriculture mécanisée : crise de production, crise de reproduction
}

\section{Abdelgalil M. Elmekki}

\section{(2) OpenEdition}

\section{Journals}

Édition électronique

URL : https://journals.openedition.org/ema/1015

DOI : 10.4000/ema.1015

ISSN : 2090-7273

\section{Éditeur}

CEDEJ - Centre d'études et de documentation économiques juridiques et sociales

\section{Édition imprimée}

Date de publication : 31 décembre 1993

Pagination : 51-70

ISSN : 1110-5097

\section{Référence électronique}

Abdelgalil M. Elmekki, «L'agriculture mécanisée : crise de production, crise de reproduction », Égypte/ Monde arabe [En ligne], 15-16 | 1993, mis en ligne le 08 juillet 2008, consulté le 07 juillet 2022. URL : http://journals.openedition.org/ema/1015 ; DOI : https://doi.org/10.4000/ema.1015

Ce document a été généré automatiquement le 7 juillet 2022.

Tous droits réservés 


\title{
L’agriculture mécanisée : crise de production, crise de reproduction
}

\author{
Abdelgalil M. Elmekki
}

\section{NOTE DE L'ÉDITEUR}

Traduit de l'anglais par François Ireton.

1 Depuis le début des années 80 tout particulièrement, le Soudan fait face à une crise sérieuse, qui se manifeste par le déclin de là production et la faillite partielle du système productif, la persistance d'un déficit de la balance courante des paiements, la paupérisation de larges fractions de la population tant rurale qu'urbaine, des processus de mobilité sociale descendante et de marginalisation et un accès de plus en plus difficile, économiquement parlant, aux produits alimentaires. Au total, les années 80 auront été celles de l'insécurité alimentaire' de la dégradation des conditions de vie, de l'instabilité politique et de l'exacerbation ces conflits.

2 De 1980 à 1985, la dépendance croissante par rapport à l'étranger, la crise alimentaire et la désintégration sociale ont aggravé les tensions politiques et conduit à une rupture de l'alliance politique au pouvoir et, en 1985, à une réorganisation du bloc dirigeant- A la fin de cette décennie, il est devenu évident que l'alliance formée sous l'hégémonie de l'ancienne bourgeoisie revenue au pouvoir était incapable de faire face à la situation générale du pays. Tout d'abord, cette alliance était trop fragile et les bases économiques des différentes fractions sociales qui la composaient avaient été ébranlées dès les années 70. Par ailleurs, l'ancienne bourgeoisie ne parvenait pas à se donner des objectifs économiques clairs et à définir une stratégie de mobilisation des ressources susceptible de favoriser le développement d'un capitalisme national. Enfin, la faiblesse de cette ancienne bourgeoisie et de l'alliance politique constituée en 1985 s'exprimait clairement dans leur incapacité à tenir compte, dans leur projet, de certains éléments des revendications issues des classes populaires - ceci sans nécessairement transformer cette alliance en y intégrant des représentants de ces dernières. 
Seule exception à ce refus d'intégrer une part des revendications populaires; la tentative, à vrai dire peu fructueuse, de limiter le pouvoir de la "fraction islamique " de la bourgeoisie, qui avait acquis une influence croissante dans les années 70 et le début des années 80 et avait fini par constituer la base économique et sociale principale du régime de Nimeyri ${ }^{1}$. Représentée par le Front national islamique (FMI), cette fraction avait consolidé elle-même sa position économique et sociale et il n'était donc guère aisé de la marginaliser politiquement. Cette évolution devait conduire au second événement majeur des années 80 que fut le coup d'État militaire, suivi d'une restructuration rapide de l'économie et de la société soudanaises.

4 Cependant, les déséquilibres économiques internes et externes, l'insécurité alimentaire, la dégradation des conditions sociales d'existence et l'instabilité politique sont les symptômes d'une crise structurelle globale qui est essentiellement une crise du secteur agraire. Cette dernière s'étend à tous les sous-secteurs de l'économie agricole : agriculture irriguée, agriculture pluviale des grandes exploitations semi-mécanisées, agriculture pluviale paysanne « traditionnelle » et élevage.

Dans le contexte de l'évolution du secteur agricole des années 70-80, les difficultés du sous-secteur semi-mécanisé apparaissent comme déterminantes dans le déclenchement de la crise agraire, et ceci pour plusieurs raisons :

- depuis le début des années 70, ce sous-secteur avait un rôle " pilote » dans l'économie tout entière ;

- il faisait l'objet d'une attention particulière de la part de l'État ;

- il était aux mains de la fraction la plus puissante de la bourgeoisie ;

- enfin, il était supposé, d'une part, contribuer le plus aux recettes d'exportation et donc à l'obtention de devises étrangères, et, d'autre part, au maintien de la sécurité alimentaire deux domaines cruciaux dans le contexte de la présente crise.

6 Dans cette étude, le concept de crise est utilisé dans un sens général aussi bien que spécifique. En général, on dit qu'un système est en crise quand il échoue à atteindre ses propres objectifs. Néanmoins, de mauvaises performances ne suffisent pas à déterminer une crise (Barker, 1984: 11-14). Plus spécifiquement, une crise se produit lorsque le système ne parvient pas à produire les ajustements internes nécessaires à sa propre reproduction et à celle des forces sociales qui bénéficient de lui. Au Soudan, le secteur agricole mécanisé oscille entre la simple difficulté à remplir sa mission (crise au sens général) et l'incapacité à se reproduire (crise au sens spécifique).

Le présent article tente d'analyser l'évolution récente de ce secteur, qui le mène d'une crise de production à une crise radicale de reproduction. On examinera tout d'abord les contextes économiques et politiques dans lesquels prend place cette évolution, puis les conséquences de ces mauvaises performances sur l'économie nationale. Le concept de sécurité alimentaire occupera ici une place centrale, puisque c'est sur ce secteur mécanisé que l'on comptait, non seulement pour assurer l'approvisionnement vivrier du Soudan, mais aussi pour combler les déficits alimentaires des pays du Moyen-Orient.

8 Les relations entre le secteur mécanisé et l'insécurité alimentaire du pays seront analysées sous trois aspects :

- l'échec du secteur à engendrer l'offre céréalière adéquate ;

- la non-solvabilité de la demande de sorgho issue d'une large majorité des consommateurs, étant donné les coûts de production de cette céréale dans ce secteur ; 
- les pressions exercées par le secteur mécanisé sur les structures paysannes de production vivrière, conduisant à leur marginalisation économique et géographique et, du même coup,

à la disparition des systèmes paysans, auparavant efficaces, de sécurité alimentaire domestique.

9 Cette étude développe trois arguments. Premièrement, la crise du sous-secteur agricole mécanisé est celle d'un modèle de développement qui doit être appréhendé dans ses relations avec les structures économiques et sociopolitiques globales. C'est la crise d'un modèle de développement capitaliste artificiel qui suppose un subventionnement étatique considérable et exerce des effets nocifs sur les autres secteurs de l'économie. Lorsque ces subventions se sont mises à décliner, les performances du sous-secteur ont fait de même et ce dernier a commencé à connaître des difficultés à se reproduire selon ses modalités de fonctionnement d'origine (dépendant des subventions).

Deuxièmement, la fraction de la bourgeoisie dont la prospérité reposait sur les performances artificielles de ce sous-secteur n'a qu'une vision étroite de ses intérêts et, contrairement à ce qui constitue la caractéristique d'une bourgeoisie agraire établie, ne se sent pas concernée par la reproduction à long terme des moyens de production auxquels elle a accès. Constitués pour leur plus grande part de marchands et de fonctionnaires d'État, les locataires des grands «domaines » mécanisés sont animés d'une mentalité purement mercantile qui se soucie bien peu de l'usage - largement irrationnel - qui est fait, dans ce système d'exploitation, des ressources naturelles. Les résultats négatifs inéluctables d'une telle insouciance se traduisent par la dégradation des écosystèmes et les faibles rendements de ces exploitations.

11 Troisièmement, l'extension et la crise du sous-secteur mécanisé ne peuvent être compris qu'en relation avec l'évolution des caractéristiques de l'État soudanais et de la nature des forces sociales dont les intérêts sont représentés au sein de ce dernier. Les correspondances que l'on peut établir entre les mesures politiques et les intérêts et pratiques des locataires de grands «domaines » mécanisés ne sont les produits ni de coïncidences ni d'une instrumentalisation de l'État par cette fraction de la bourgeoisie, ni de simples opérations de «lobbying». Elles sont plutôt le produit de rapports objectifs entre l'État et cette puissante fraction de la bourgeoisie.

Extension et performances de l'agriculture mécanisée

12 Le secteur agricole est l'épine dorsale de l'économie soudanaise. Il contribue pour un tiers au produit intérieur brut et regroupe $80 \%$ de la population active. L'agriculture mécanisée fournit à peu près la moitié des emplois comme des revenus de ce secteur (ILO, 1987: 49). Ce qui renforce encore l'importance de ce sous-secteur, c'est qu'il assure $60 \%$ de la production nationale de sorgho. De plus, il constitue le point de convergence principal des capitaux accumulés au Soudan, puisqu'il y présente les opportunités d'investissements agricoles privés de loin les plus lucratifs.

13 C'est au début des années 1940 que l'agriculture mécanisée est apparue au Soudan, l'État colonial ayant projeté d'augmenter la production céréalière pour approvisionner aussi bien le marché national que celui d'autres colonies britanniques². Il y a, dans l'histoire de la place et des fonctions du sous-secteur mécanisé agricole au sein de l'économie soudanaise, trois points d'inflexion:

- l'adoption, au début des années 50, d'objectifs d'extension massive des exploitations agricoles de grande taille utilisant le travail salarié, deux caractéristiques indispensables au développement de la mécanisation à grande échelle; 
- la création, en 1968. de la « Mechanized Farming Corporation » (MFC) destinée à gérer le sous-secteur mécanisé et à coordonner l'allocation des fonds avancés à ce dernier par l'Association internationale de développement ;

- la mise en œuvre de la stratégie dite du breadbasket dans le milieu des années $70^{3}$. L'adoption, dans les années 50, d'une organisation de la production agricole sur la base de grandes exploitations distribuant des salaires a fixé les caractéristiques sociales du sous-secteur et en a fait le noyau du développement capitaliste au Soudan. La création de la MPC - et l'apport de capitaux étrangers que sa mise en place supposait - puis, .plus tard, l'adoption de la stratégie du breadbasket ont donné au sous-secteur l'impulsion nécessaire à une expansion rapide. A ces deux occasions, celui-ci a bénéficié d'un large soutien financier étranger et s'est vu attribuer d'importants privilèges et concessions foncières par l'État lui-même. Parce qui apparaît comme une ironie de l'Histoire, ces avantages, qui ont stimulé le développement du sous-secteur, ont plus tard été à l'origine de certaines de ses difficultés les plus sévères, comme on le montrera ultérieurement.

Ces mesures politiques et avantages se traduisent, dès la fin des années 60 , par une très forte extension horizontale du sous-secteur. La superficie totale occupée par la production mécanisée passe de 12.000 feddans en 1943-44 à un million en 1969-70; elle décuple entre cette dernière date et l'année 1988-89 (Al-Sheikh, 1990). D'énormes concessions foncières et subventions sont attribuées par l'État, à travers la MFC et la Banque agricole du Soudan (BAS). D'autres facilités sont également accordées au soussecteur dans le cadre de la stratégie du breadbasket, qui place la mécanisation de l'agriculture en tête de toutes les priorités sectorielles dans la perspective de la transformation $d u$ Soudan en «grenier du Moyen-Orient». En conséquence, l'agriculture mécanisée s'étend rapidement aux terres marginales plus fragiles ainsi qu'aux surfaces déjà cultivées en sorgho et en millet dans le cadre de l'agriculture paysanne de subsistance (Al-Hassan, 1985: 373). Les résultats d'une semblable extension sont patents en ce qui concerne la dégradation des écosystèmes, la baisse des rendements et les transformations des systèmes paysans de production et de consommation alimentaires (ILO, 1987:54).

L'agriculture mécanisée était supposée participer au développement national à travers l'accomplissement des objectifs suivants :

- assurer la sécurité alimentaire nationale ;

- contribuer à la stabilité - voire aux bénéfices - de la balance des paiements en produisant du sorgho, du sésame et du coton pour l'exportation;

- élargir l'offre d'emploi rural ;

- apporter une contribution positive au développement économique et social global des communautés rurales situées à la périphérie des grandes exploitations mécanisées.

Chacun de ces objectifs s'est vu attribuer un poids différent dans les plans de développement successifs. Jusqu'au milieu des années 70 , l'objectif dominant a été la réponse du sous-secteur mécanisé à la demande intérieure de sorgho bon marché. Les objectifs liés à cette politique étaient les suivants :

- la main-d'œuvre paysanne traditionnellement productrice de sorgho (destiné à l'autoconsommation et, en cas de surplus, à la commercialisation) étant ainsi libérée de cette occupation, la réorienter vers le sous-secteur irrigué producteur de coton (sous forme de main-d'œuvre saisonnière) et vers la production de cultures commerciales (graines oléagineuses notamment) ${ }^{4}$; 
- allouer à ces mêmes cultures les terres consacrées au sorgho dans le cadre de l'agriculture paysanne ;

- approvisionner en céréales bon marché les zones urbaines pour faire baisser, ou du moins stabiliser, les salaires versés par les secteurs urbains de l'économie. ressources - naturelles, humaines et financières - mobilisables par le sous-secteur mécanisé, qui est ainsi entré dans une phase d'intenses difficultés. L'État a cessé de le subventionner substantiellement et ceci, non à la suite de décisions politiques, mais de l'impossibilité de rassembler les fonds nécessaires. De plus, toutes les terres raisonnablement utilisables dans le cadre de l'expansion horizontale ayant été mises en culture, la productivité moyenne et bientôt la production elle-même se sont mises à décliner, par épuisement des terres anciennement cultivées et adjonction de terres marginales toujours moins fertiles. Bref, les conditions favorables au développement du sous-secteur s'étant transformées dans les années 80 , il a cessé d'être ce domaine privilégié de projets et d'investissements lucratifs qu'il était encore dans les années 70 . Actuellement, les bénéfices potentiels de tels investissements sont si réduits que ce sous-secteur ne présente plus aucun avantage comparatif (ILO, 1987; 67).

Une série d'interviews menées par l'auteur en mai 1990 révèle qu'en nombre croissant, les locataires de grands « domaines » mécanisés ont renoncé à renouveler leurs baux. La plupart, en effet, désinvestissent du secteur mécanisé et se tournent vers le commerce et les transports, tout en tentant par ailleurs de réduire au minimum le coût des travaux agricoles sur les domaines qui leur restent, en diminuant par exemple le nombre de labours, de sarclages et de désherbages. Ainsi, les stratégies à courte vue adoptées pour faire face au déclin des rendements et des revenus tirés de cette forme d'agriculture ne font, en fait, que renforcer les facteurs de son déclin, en réduisant encore davantage la fertilité des sols, les rendements et finalement la rentabilité économique de ce système. Si les comportements actuels des grands locataires persistent, les grosses difficultés que connaît le sous-secteur s'étendront à sa capacité de reproduction elle-même. Le tableau 1 décrit les performances globales de l'agriculture mécanisée depuis le début des années 70, caractérisée par, les fortes fluctuations de la production et le déclin des rendements. Comparativement à la petite agriculture paysanne, les performances de l'agriculture mécanisée sont dérisoires au regard des subventions et concessions foncières qu'elle reçoit : si ses rendements sont meilleurs durant les cinq ans suivant la première mise en culture, ils déclinent ensuite nettement. L'épuisement des terres et l'impossibilité de poursuivre l'expansion horizontale de ce sous-secteur sont donc les principaux responsables du déclin global des rendements de l'agriculture soudanaise dans les années 80 . Les bonnes performances de l'agriculture mécanisée durant les années 70 , n'étaient dues qu'à son extension sur des terres arables vierges.

Tableau 1 : Production de sorgho dans le secteur mécanisé (1970/71 - 1989/90. Superficie en milliers de feddans, production en milliers de tonnes, rendement en $\mathrm{kg} / \mathrm{feddan}$ )

\begin{tabular}{|l|l|l|l|}
\hline Saison & Superficie & Production & Rendement \\
\hline \hline $1970 / 71$ & 2.014 & 670 & 333 \\
\hline
\end{tabular}




\begin{tabular}{|c|c|c|c|}
\hline $1971 / 72$ & 2.018 & 681 & 337 \\
\hline $1972 / 73$ & 1.751 & 442 & 253 \\
\hline $1973 / 74$ & 2.413 & 823 & 341 \\
\hline $1974 / 75$ & 2.952 & 751 & 254 \\
\hline $1975 / 76$ & 3.362 & 1.032 & 307 \\
\hline $1976 / 77$ & 3.369 & 1.047 & 311 \\
\hline $1977 / 78$ & 3.497 & 1.203 & 344 \\
\hline $1978 / 79$ & 2,814 & 1.066 & 379 \\
\hline $1979 / 80$ & 3.434 & 779 & 226 \\
\hline $1980 / 81$ & 5.532 & 1.214 & 219 \\
\hline $1981 / 82$ & 5.305 & 2.150 & 405 \\
\hline $1982 / 83$ & 5,502 & 1.185 & 215 \\
\hline $1983 / 84$ & 4.534 & 1.179 & 260 \\
\hline $1984 / 85$ & 7.335 & 389 & 53 \\
\hline $1985 / 86$ & 5.315 & 2.331 & 438 \\
\hline $1986 / 87$ & 9.747 & 1.806 & 185 \\
\hline $1987 / 88$ & 5.830 & 853 & 146 \\
\hline $1988 / 89$ & 9.747 & 3.317 & 340 \\
\hline $1989 / 90$ & 5.527 & 853 & 154 \\
\hline
\end{tabular}

Source : ILO, 1987, p. 66 ; Ministère des Finances, Economic Survey; Ministère de l'Agriculture. Département des statistiques et de l'économie agraires.

19 L'expansion horizontale incontrôlée qu'ont connue ces années a été facilitée par l'atmosphère très favorable à ces opérations qu'engendrait l'adhésion à la stratégie du breadbasket. Les subventions supplémentaires accordées à ce sous-secteur, jointes à l'obtention de plus forts revenus d'exploitation qu'apportait la réorientation des débouchés de la production mécanisée, passant du marché intérieur aux marchés d'exportation, ont poussé l'expansion horizontale du sous-secteur mécanisé à son taux maximal. A en croire O'Brien, les transformations opérées dans les années 70 ont créé toutes les conditions de grande vulnérabilité des populations rurales à la famine 
(O'Brien, 1985 ; 24). La nouvelle orientation vers l'exportation, le déclin global des rendements et l'augmentation - qui leur est liée - du prix du sorgho sur les marchés locaux, rendent éminemment contestable la contribution du sous-secteur mécanisé au maintien de la sécurité alimentaire de la population.

Tableau 2 : Part de l'agriculture mécanisée dans la surface cultivée totale (a), la surface cultivée en sorgho (b) et la production totale de sorgho (c) (\%).

\begin{tabular}{|c|c|c|c|}
\hline & (a) & (b) & (c) \\
\hline $1975 / 76$ & 27 & 53 & 57 \\
\hline $1976 / 77$ & 27 & 50 & 59 \\
\hline $1977 / 78$ & 24 & 47 & 45 \\
\hline $1978 / 79$ & 23 & 46 & 52 \\
\hline $1979 / 80$ & 24 & 43 & 59 \\
\hline $1980 / 81$ & 27 & 50 & 66 \\
\hline $1981 / 82$ & 34 & 60 & 66 \\
\hline $1982 / 83$ & 32 & 57 & 61 \\
\hline $1983 / 84$ & 36 & 60 & 60 \\
\hline $1984 / 85$ & 33 & 57 & 36 \\
\hline $1985 / 86$ & 41 & 60 & 61 \\
\hline $1986 / 87$ & - & 71 & 63 \\
\hline $1987 / 88$ & - & 70 & 65 \\
\hline $1988 / 89$ & - & 73 & 75 \\
\hline $1989 / 90$ & - & 63 & 56 \\
\hline
\end{tabular}

Source : ILO, 1987, et Ministère des Finances, Economic Survey.

20 L'examen des performances globales de ce dernier durant les années 70 soulève nombre de questions concernant sa rentabilité économique, sa contribution à l'aggravation des problèmes sociaux et son impact écologique. Jusqu'à récemment, l'agriculture mécanisée apparaissait cependant encore comme apportant une meilleure solution globale au problème de la production de sorgho que ne le faisait le secteur agricole-»traditionnel»; la simple raison en était la possibilité d'expansion horizontale de ce sous-secteur. Le tableau 2 ci-dessus montre que ce dernier couvrait, 
au début des années 80 , à peu près le tiers de la surface cultivée du Soudan et la moitié de la surface nationale emblavée en sorgho et contribuait pour les deux tiers à la production nationale de cette denrée. Cependant, cette proportion ne se reflétait pas aussi clairement dans la contribution de ce sous-secteur à la sécurité alimentaire du pays, dans la mesure où la production de cette céréale pour l'exportation avait pris le pas sur la production pour le marché intérieur, ceci dans le contexte de la stratégie du breadbasket.

Organisation de la production et caractéristiques sociopolitiques des grands locataires

21 Cinq types d'acteurs sont concernés par l'organisation de la production dans le soussecteur d'agriculture mécanisée: ce sont l'État, les locataires et/ou possesseurs de capitaux, les régisseurs d'exploitation et les travailleurs agricoles. Les rapports entre ces différentes catégories sont importants à prendre en considération pour comprendre les modes de répartition du surplus et l'implication de ces catégories dans le processus de production et le sort que leur réserve la crise actuelle. L'État est le propriétaire légal. Il loue la terre aux preneurs de bail moyennant une rente versée par ces derniers. De plus, il consent aux grands locataires des facilités de crédit en vue de l'achat d'équipements et de la constitution d'un capital d'exploitation, cela par le biais de la MFC et de la BAS. Il consent également aux locataires des taux de change d'encouragement pour leurs achats d'équipements et d'intrants importés. Enfin, bien souvent, l'État assure le dédommagement de la MFC ou de l'ABS en cas de nonremboursement de la part des grands locataires.

A chacun de ces derniers est attribué un lot de terres d'une superficie moyenne de 1.000 à 1.500 feddans et, théoriquement du moins, personne ne peut se voir attribuer plus d'un lot. Ces locataires sont la plupart du temps de riches marchands ou des fonctionnaires d'État. Ils investissent eux-mêmes dans la mise en valeur du domaine ainsi loué ou s'engagent, pouf ce faire, dans des arrangements à part de fruit avec des possesseurs de capitaux. En d'autres termes, les locataires et les détenteurs de capitaux ne forment que rarement une seule et même catégorie. Il existe trois formes d'arrangement entre ces deux catégories. Soit le locataire peut céder le bail à un financier en échange d'une somme fixe supérieure au montant de ce bail (le locataire n'est alors qu'un intermédiaire). Soit le locataire et le financier montent conjointement un projet, l'un des partenaires assurant le paiement de la rente due à l'État et faisant bénéficier le projet des avantages financiers que l'État lui consent, l'autre partenaire amenant le gros du capital d'exploitation et parfois sa capacité à gérer le domaine ; en principe, le locataire reçoit alors de 10 à $25 \%$ de la récolte, sans considération des frais d'exploitation ni des rendements. Enfin, un troisième arrangement est possible: le locataire paie la rente et apporte, outre les prêts avantageux consentis par l'État, une partie du capital d'exploitation. Le financier, pour sa part, amène le solde du capital d'exploitation et ses capacités personnelles de gestion. Dans ce dernier cas, tous les risques et profils sont partagés selon certaines proportions variables.

Le premier type d'arrangement, qui est le plus répandu, a de sérieuses répercussions sur les performances globales du sous-secteur mécanisé. Le locataire ne se sent en effet pas concerné par le caractère approprié ou inapproprié des méthodes de cultures mises en œuvre, dans la mesure où, quels que soient les résultats d'exploitation, il touchera en espèces la même somme: celle qui a été fixée en échange de la sous-location consentie aux détenteurs de capitaux. De même, le locataire comme le détenteur de capitaux ne se sentent guère concernés par la préservation à moyen ou long terme de la 
fertilité de la terre, car il leur sera toujours loisible de prendre en location, après quelques années, un lot de terres non encore cultivées, ce qui leur est rendu possible par les très faibles investissements qu'il font dans la terre elle-même en vue de la reproduction de sa fertilité et par l'absence de construction, sur ces terres, de bâtiments d'exploitation.

La seconde forme d'arrangement n'est guère plus favorable au maintien de la fertilité et des rendements, dans la mesure où locataires comme financiers ont intérêt à minimiser les frais d'exploitation en réduisant les opérations de labour et de désherbage, de façon à ce que les coûts constituent la plus faible part possible des revenus, dont locataires et financiers se partagent en moyenne 75 à 90 \% (Al-Amin, $1989 ; 219)$.

Le résultat majeur de ces arrangements de sous-locations ou à part de fruit dans le sous-secteur mécanisé réside dans le fait que, quelles que soient les modalités concrètes des relations entre les partenaires, "se forme un groupe étroit de financiers qui monopolisent de fait (à travers le partenariat de chacun d'eux avec de nombreux locataires) la plus grande part des exploitations d'agriculture pluviale mécanisée et qui tiennent en main - et manipulent - aussi bien la production que la commercialisation des denrées agricoles principales ». (Karsani, 1982:14). On cite l'exemple d'un financier devenu, à lui seul, partenaire dans les opérations d'investissement et de gestion de 16 lots de terres (soit 24.000 feddans), dans le périmètre agricole de Habila (Karsani, op. cit.). Il est clair que la possession de capitaux n'est pas nécessaire à l'acquisition de baux de grands domaines mécanisés. Des fonctionnaires et des membres de l'armée ou de la police utilisent fréquemment leurs relations avec les sphères du pouvoir pour obtenir ces baux et conclure des arrangements "spéciaux» avec les financiers. En fait, l'obtention de baux devient un bénéfice statutaire de hauts fonctionnaires et d'officiers de l'armée et de la police partant en retraite.

Les financiers, qui constituent la catégorie pivot des acteurs impliqués dans ce soussecteur agricole, sont presque toujours des marchands urbains qui pratiquent le commerce intérieur et extérieur du sorgho. Ils constituent la fraction la plus puissante de la bourgeoisie, celle qui contrôle la production et la circulation des biens alimentaires de première nécessité au niveau intérieur. Durant les années 80 , ces activités sont devenues le domaine favori et « réservé » de marchands associés avec les institutions financières islamiques, telle la "Faysal Islamic Bank », qui s'est spécialisée, depuis sa fondation en 1978, dans le contrôle de l'approvisionnement en sorgho. La concentration de grandes étendues de terres entre les mains de quelques détenteurs de capitaux s'est renforcée par la tendance croissante à l'attribution de baux à des « compagnies » nationales et étrangères, tendance qui est le produit de l'adoption de la stratégie du breadbasket des années 70. Alors que les lots accessibles à des individus couvrent de 1.000 à 1.500 feddans chacun, ceux qui sont dévolus aux compagnies peuvent aller jusqu'au million de feddans, mais leur superficie tourne en général autour de 10.000 à 40.000 feddans.

$\mathrm{Ni}$ les locataires ni les financiers ne sont cependant impliqués directement dans l'organisation des opérations de production agricole. Dans tous les cas, ces dernières sont supervisées par des régisseurs salariés, qui planifient le travail et veillent à son exécution. C'est pour cette raison que grands locataires et financiers sont baptisés " agriculteurs d'occasion ", car ils n'entendent la plupart du temps rien aux travaux et techniques agricoles. Leur profession principale elle-même contredit le principal 
critère d'attribution des baux, qui veut que le locataire soit agriculteur au sens propre du terme. On considère habituellement que cette incompétence et cet absentéisme constituent l'une des principales raisons de l'impact tout à fait nocif de ce type d'exploitation mécanisée.

La composition sociale des locataires et financiers fournit donc un des éléments d'explication de l'émergence de la crise du sous-secteur mécanisé. Ces acteurs ne voient en effet dans ce type d'agriculture qu'une occasion de «faire des affaires » dans la plus pure logique marchande. Ce sous-secteur n'est donc qu'un moyen de profits rapides, réalisés en fait au détriment de la reproduction à long terme des conditions «naturelles » de la production et d'abord de la fertilité de la terre et des équilibres de l'écosystème, toutes choses qui constituent le cadet des soucis de ces protagonistes d'une économie " prédatrice ".

29 Le déploiement de cette logique marchande a été toléré, puis admis par l'État. La MFC n'a pas été capable de contrôler le processus d'expansion horizontale et de limiter le laxisme dont il a été fait preuve vis-à-vis des infractions à la législation visant à limiter les dégâts écologiques causés par ce type d'agriculture. La logique marchande s'est avérée politiquement plus puissante que les logiques agronomiques, techniques et de préservation des intérêts collectifs.

Mesures politiques vis-à-vis de l'expansion incontrôlée et des pratiques agricoles nocives

30 Les mesures politiques adoptées à l'égard des différents secteurs agricoles ont été extrêmement déséquilibrées. Elles ont été en général déterminées par les rapports de pouvoir, variant selon les blocs d'alliance parvenus à la direction du pays. Le secteur agricole mécanisé a été celui au sein duquel la fraction la plus puissante de la bourgeoisie a trouvé, depuis le début des années 50, les moyens de son ascension sociale et de sa reproduction. C'est autour de ce même secteur qu'ont convergé les intérêts matériels des hauts fonctionnaires de l'État et de la bourgeoisie marchande, de sorte que les premiers sont passés du statut de simples instruments de la classe économiquement dirigeante à celui de membres à part entière de cette dernière.

31 Cette transition est capitale pour expliquer le préjugé favorable dont a bénéficié le sous-secteur mécanisé de la part de l'État. En fin de compte, ce dernier est lui-même devenu le principal promoteur de la logique marchande qui a présidé à l'expansion de ce type d'agriculture, au lieu de veiller à la préservation des équilibres nécessaires à la reproduction à long terme des activités agricoles; ceci en dépit des mesures officielles de préservation de ces équilibres. L'attribution massive de subventions - visibles ou invisibles - qui est ainsi devenue une condition de la reproduction du sous-secteur en question, a également été un facteur déterminant du malaise de ce dernier, lorsque l'Etal soudanais s'est vu contraint de réduire ces subventions, dans le cadre du processus de « libéralisation » qui caractérise son actuelle politique économique.

Les pratiques nuisibles qui sévissent dans l'agriculture mécanisée renvoient aux modes d'accumulation mis en œuvre par les grands locataires et les financiers avec la " complicité » de l'État. Une expansion horizontale effectuée à un tel rythme n'a en effet été possible, dès ses débuts, 'qu'en l'absence de tout contrôle véritable de la part de l'État. Les subventions substantielles venant de ce dernier et le laxisme dont il a fait preuve ont contribué notoirement à faire de l'agriculture mécanisée l'activité économique la plus attirante et de l'expansion horizontale le moyen le plus simple de s'enrichir rapidement. La législation sur les baux n'a fait qu'aggraver le caractère 
chaotique de cette expansion et l'inadéquation des pratiques culturales et de gestion. Ainsi, la possibilité de contracter des baux de cinq ans seulement a encouragé à l'exploitation purement "minière " d'un sol que l'on abandonne sitôt épuisé, pour prendre à bail un autre lot de parcelles « fraîches ». Un tel mode de fuite en avant des exploitations s'est poursuivi jusqu'au début des années 80 sous les auspices de la MFC, jusqu'à ce qu'il ne reste pratiquement plus de terres vierges susceptibles de permettre la poursuite du processus. A ce moment-là, le développement de l'agriculture mécanisée a pris deux directions parallèles. D'une part, elle a été étendue aux terres marginales situées dans les zones de pluviométrie annuelle inférieure à $300 \mathrm{~mm}$. D'autre part, les locataires, dans l'impossibilité d'opérer un tel transfert, se sont mis à surexploiter leurs anciennes terres et à n'y plus pratiquer que la monoculture du sorgho sans pour autant prendre enfin les mesures nécessaires à la restauration des sols (ou du moins, à l'arrêt de leur dégradation). Dans les deux cas, ces pratiques se sont avérées désastreuses pour la production et l'écosystème et ont contribué clairement à la désertification par destruction quasi complète du couvert végétal et accélération de l'érosion (Sudannow, janvier 1985:10).

L'expansion horizontale, approuvée par l'État, a conduit non seulement à la chute des rendements et à la dégradation des sols, mais aussi au déplacement forcé des petits paysans et agro-pasteurs "traditionnels». Ces derniers ont été repousses vers les terres marginales et parfois vers des zones pratiquement inaccessibles, à sols pauvres n'autorisant que de très faibles rendements. Quant aux pasteurs, ils n'ont eu d'autre recours que le surpâturage des terrains de parcours résiduels. Dans les deux cas, c'est la sécurité alimentaire de ces populations qui s'est trouvée directement menacée.

Hormis l'expansion horizontale, trois autres types de pratiques propres au sous-secteur mécanisé ont contribué à engendrer la situation actuelle. D'abord, aucune rotation culturale n'a vraiment été suivie, bien qu'en théorie l'on aurait dû faire suivre une culture annuelle de sorgho par une autre de sésame, de coton, de tournesol, puis d'une troisième année de jachère. Récemment, il a été proposé d'inclure dans les rotations certaines cultures légumières, mais cette suggestion n'a jamais été appliquée par les grands locataires. En fait, ces derniers, comme les financiers, ont toujours préféré s'en tenir à la monoculture du sorgho, culture la moins exigeante et la plus profitable. Cette monoculture a contribué bien évidemment à la dégradation des sols et à la chute des rendements.

Ensuite, la logique purement marchande n'a guère favorisé l'adoption de techniques culturales appropriées au type de cultures pratiquées dans un tel écosystème. Le sarclage, opération cruciale pour le maintien des rendements, est souvent effectué de manière défectueuse et pas aussi souvent que l'exigerait la préservation à long terme de la fertilité. Ceci n'est qu'un exemple supplémentaire de stratégie de minimisation des coûts et de maximisation des profits en harmonie avec les modes de fonctionnement à courte vue du sous-secteur : courte durée des baux et agriculture minière. Pratiques qui, là encore, conduisent à la réduction des éléments organiques et à la prolifération des insectes, principaux responsables, certaines années, de très bas rendements.

Enfin, le couvert végétal et forestier a été totalement éliminé lors des opérations de défrichement préalables à la mise en culture, l'érosion éolienne des sols appauvris faisant le reste. Il se peut d'ailleurs que la baisse durable de la pluviométrie soit la conséquence, tout autant que la cause, de la disparition de cette couverture végétale. Il 
semble en effet fort probable que "lorsque le sol est privé de sa végétation, les taux de rayonnement augmentent, ce qui affecte l'intensité des températures, des vents et des précipitations (Sudan now, janvier 1985 : 10). L'État s'est révélé impuissant à imposer des mesures concrètes de protection de l'environnement et la MFC n'a pas mieux réussi à cet égard, ce qui n'a pu que servir les intérêts des locataires comme ceux de la haute bureaucratie d'État, dont on a vu les liens qu'elle entretenait avec ces derniers.

Tableau 3 : Taux de croissance annuels du secteur agricole soudanais (\%)

\begin{tabular}{|l|l|l|l|}
\hline $1982 / 83$ & -8.9 & $1986 / 87$ & 2.0 \\
\hline \hline $1983 / 84$ & -4.6 & $1987 / 88$ & -12.3 \\
\hline \hline $1984 / 85$ & -122 & $1988 / 89$ & 27.3 \\
\hline $1985 / 86$ & 9.9 & $1989 / 90$ & -8.1 \\
\hline \hline \multicolumn{2}{|l|}{ Moyenne : 0.3} & \\
\hline
\end{tabular}

Source : Ministère des Finances, Economic Survey, 1989/90, p. 22.

L'ensemble de ces processus a donc conduit à la présente crise alimentaire comme à la pénurie de devises étrangères que certaines cultures sont censées rapporter. Il est clair que le Soudan est menacé de famine non seulement du fait des fluctuations pluviométriques, mais encore du fait des modes d'organisation et de fonctionnement de la production agricole et de la distribution des bénéfices dans ce domaine, qui restreignent l'offre de produits alimentaires et limitent leur accessibilité. L'agriculture soudanaise tout entière a ainsi enregistré, durant les années 80 , une croissance négative et l'on est passé d'une période de stagnation à une période de déclin en chiffres absolus de volume produit (et pas simplement de volume per capita). Le tableau 3 ci-dessus montre l'ampleur du phénomène durant cette période.

L'opinion publique, préoccupée par la crise alimentaire, a d'autant plus focalisé son attention sur les contre-performances du sous-secteur mécanisé qu'il était censé faire tampon face aux risques de famine. L'État, quant à lui, s'est beaucoup plus préoccupé des conséquences de ces contre-performances sur le déclin des exportations que de la baisse des disponibilités alimentaires intérieures qu'elles entraînaient. Il est clair qu'il nourrit les plus grandes craintes face à la diminution de la contribution du sous-secteur agricole mécanisé au produit intérieur brut et à la disproportion entre le niveau de cette contribution et la part considérable des fonds publics que le subventionnement du sous-secteur absorbe encore. Le tableau 4 montre l'évolution de cette contribution durant les années 80 ; elle n'a jamais dépassé les 6,3\% durant cette période, et les exportations de sorgho n'ont pas excédé $17 \%$ de la valeur totale des exportations.

Tableau 4 : Contribution de l'agriculture mécanisée au PIB en prix constant et en pourcentage

\begin{tabular}{|l|l|l|l|l|l|l|}
\hline $1981 / 82$ & 4.8 & $1985 / 86$ & 4.8 \\
\hline
\end{tabular}




\begin{tabular}{|l|l|l|l|}
\hline $1982 / 83$ & 2.2 & $1986 / 87$ & 2.2 \\
\hline \hline $1983 / 84$ & 2.3 & $1987 / 88$ & 6.3 \\
\hline \hline $1984 / 85$ & 1.2 & $1988 / 89$ & 2.4 \\
\hline
\end{tabular}

Source : Ministère des Finances, Economic Survey 1989/90, p. 17.

Mais ces préoccupations étatiques vis-à-vis de l'agriculture mécanisée ne se sont traduites par aucune mesure concrète de politique agricole. La seule exception, dans ce domaine, est constituée par la mise en place, en 1986, de la "Stratégie pour le développement de l'agriculture pluviale", lancée et financée par l'Association internationale pour le développement. Cette stratégie visait aussi bien le sous-secteur d'agriculture pluviale paysanne «traditionnelle » que celui de l'agriculture mécanisée. Néanmoins, dans la droite ligne des orientations antérieures, l'attention s'est concentrée bien davantage sur ce dernier que sur le premier, pourtant crucial dans la mise en œuvre de toute stratégie efficace de sécurité alimentaire. Le plan proposé se donnait cinq objectifs :

- l'auto suffisance alimentaire du pays et une contribution à la sécurité alimentaire de la région (Afrique de l'Est);

- la production de surplus agricoles exportables ;

- l'augmentation des revenus des producteurs et le soutien à l'emploi de la population rurale ;

- la conservation et la réhabilitation des ressources naturelles ;

- enfin l'encouragement de l'investissement privé et des initiatives visant à augmenter la production, par l'installation d'infrastructures et de services et l'adoption de mesures appropriées (Ministère des Finances, 1985 : 5-6).

Un certain nombre de remarques s'imposent concernant ces objectifs. Tout d'abord, mis à part le quatrième, aucun d'eux ne paraît nouveau pour ce qui louche au soussecteur mécanisé. Ensuite, aucune attention particulière n'est portée sur le contexte social de la production agricole pluviale et les recommandations sont essentiellement d'ordre technique et partiellement économique. Par ailleurs, cette "stratégie » suppose, sans discussion, le maintien de l'appui substantiel de l'État, à travers des mesures de dégrèvements fiscaux et un système de fixation de taux de change et de prix avantageux. Enfin, si, pour la première fois, il est affirmé que le sorgho ne constitue pas une culture d'exportation de grand rapport, les implications politiques d'une telle affirmation ne sont pas développées.

41 Finalement, la quasi-totalité des mesures proposées visent la réhabilitation de ce qui s'est dégradé durant les quinze ou vingt années précédentes, mais les racines structurelles de cette dégradation ne sont pas analysées. Dans ces conditions, l'on peut sérieusement s'interroger sur la viabilité d'un plan de réhabilitation mis en place dans un contexte inchangé : celui, précisément, qui a abouti à la crise à laquelle le plan entend remédier. Il est enfin clair que, dans le cadre de ce plan, l'État n'est nullement en position de pouvoir faire pièce aux intérêts à court terme de la bourgeoisie agrocommerciale, alors que la préservation des intérêts collectifs à long terme devrait requérir le déploiement de toutes les forces de résistance mobilisables.

Conclusion 


\section{BIBLIOGRAPHIE}

'Abd al-Mun'im 0. E. A., « Marketing and Pricing Policies of Mechanized Farming Crops » (paper presented to the Conference on Mechanized Farming, Gedaref), 1990.

Adam F. H., « Agrarian Relations in Sudanese Agriculture: A Historical Review », Sudan Journal of Development Research, 1,2, 1977.

Awad M. H., Why is the breadbasket empty?, Khartoum, DSRC, seminar series bulletin, $\mathrm{n}^{0} 40,1983$.

Barker J., The Politics of Agriculture in Tropical Africa, London, SAGE, 1984.

Barnet T. and Abd al-Karim A. (eds.), Sudan. Capital and Transformation, London, Groom Heim, 1988.

El-Amin A., «Land Allocation for Mechanized Farming », paper presented to the Conference on Mechanized Farming, Gedaref, 1990.

El-Amin G. M., «Production and Marketing of Sorghum », in Institute of Social Studies Advisory Service, Food Security in the Sudan. The Hague, 1989, pp. 214-255.

Égypte/Monde arabe, 15-16 | 1993 
El-Hassan A. M., The State and the Development of Capitalism in Agriculture in Sudan, University of East Anglia, Ph. D. Dissertation.

Al-Karib J., The Political Economy of Sorghum Production, University of Khartoum, DSRC Diploma Dissertation, 1990.

Elmekki A.M., The Development of Capitalism in Gedaref, University of Khartoum, Department of Political Science, B. Sc. Dissertation, 1978.

Elmekki A.M., Peasants and Capital in the Sudan, University of Toronto, Ph.D. Dissertation, 1985.

Elmekki A.M., From Self-Sufficiency to PL 480 : The Political Economy of Food Production and Starvation in the Sudan, University of Maryland, Department of Government and Politics, Discussion Paper, 1988.

El-Sheikh A. et al., " The Economic Importance of the Rainfed Agricultural Subsector in Sudan », paper presented to the Conference on Mechanized Farming, Gedaref, 1990.

El-Sheikh A., « Production Policies in Mechanized Farming », paper presented to conference on Mechanized Farming, Gedaref, 1990.

Institute of Development Studies, Food Security Study : Phase I, University of Sussex, Sussex, 1988.

Ilo (International Labour Organization), Employment and Economic Reform: Towards a Strategy for the Sudan, Geneva, 1987.

Kursani I., The Dynamics and Limits of Capitalist Development in Sudanese Agriculture, University of Khartoum, DSRC Discussion Paper, $\mathrm{n}^{0} 17,1982$.

Mahmud F. B., The Sudanese Bourgeoisie, London, Zed, 1984.

Ministry of Finance and Economic Planning, Strategy for Development of Rainfed Agriculture, Khartoum, 1986.

Ministry of Finance and Economic Survey, various issues.

O'Brien J. J., Agricultural Labour and Development in the Sudan, University of Connecticut, Ph. D. Dissertation, 1980.

O'Brien J. J., « Sudan : an Arab breadbasket? », Merip Report, 1981, 99.

O'Brien J. J., « The Political Economy of Capitalist Agriculture in the Central Rainlands of the Sudan ", Labour, Capital and Society, 16,1,1983, pp. 23-32.

O'Brien J. J., « Sowing the Seeds of Famine: The Political Economy of Food Deficits in Sudan », Review of African Political Economy, 33,1985.

Shepherd A., "Capitalist Agriculture in the Sudan's Dura Prairies », Development and Change, 14, 1983, pp. 297-320.

Sudan Now, various issues.

Wohlmuth K., The Development Perspectives of the Democratic Republic of the Sudan: The Limits of the Breadbasket Strategy, München, Bremen University, 1983.

The World Bank, Sudan: Towards an Action Plan for Food Security, Washington, D.C., IBRD, 1990. 


\section{NOTES}

1. Par « fraction islamique de la bourgeoisie », je désigne ces nouveaux groupes sociaux apparus vers le milieu des années 70 , qui sont en relation économique et financière étroite avec les institutions bancaires islamiques du type de la Faysal Islamic Bank. 2. Pour un rappel historique, voir Elmekki, 1978, ADAM, 1977, et O'Brien, 1980 et 1983. 3. Pour une description détaillée et une analyse de cette stratégie, voir O'Brien, 1981, Wohlmuth, 1983, et Awad, 1983.

4. Pour une analyse conceptuelle et empirique de ce processus ainsi que de ses conséquences socio-politiques, voir Elmekki, 1985.

INDEX

Mots-clés : Soudan

\section{AUTEUR}

ABDELGALIL M. ELMEKKI

Université de Khartoum 